Revista de Filosofía

Volumen 66, (2010) 25 - 44

\title{
EL UNIVERSAL DE PLURIVERSO
}

\author{
José Tomás Alvarado Marambio \\ Pontificia Universidad Católica de Valparaíso \\ jose.alvarado.m@ucv.cl
}

\begin{abstract}
Resumen
Este trabajo presenta la idea de un único pluriverso en vez de una pluralidad de mundos posibles como explicación de los hechos modales. Primero, se expone la idea y motivaciones de una oración de pluriverso en el contexto de una teoría lingüística de la modalidad. Luego se defiende una concepción de los hechos modales dependiente de universales, en la que los mundos posibles deben ser entendidos como universales estructurales máximos. Aquí, el universal de pluriverso es una única propiedad que determina la totalidad del espacio modal. Cada forma en que el mundo podría ser es representada en el universal de pluriverso como una cláusula disyuntiva en el universal. Finalmente, el trabajo presenta una dificultad fundamental para el universal de pluriverso como explicación metafísica de la modalidad, pues éste parece requerir un dominio previo de objetos actuales y meramente posibles.
\end{abstract}

Palabras Clave: modalidad, metafísica de la modalidad, mundos posibles, pluriverso, universales.

Abstract

This work presents the idea of a unique pluriverse instead of a plurality of possible worlds as an explanation of modal facts. First we state the idea and motivations of a pluriverse sentence in the context of a linguistic theory of modality. Then we defend a conception of modal facts dependent on universals in which possible worlds should be understood as maximal structural universals. Here the pluriverse universal is one single property determining the totality of the modal space. Each way the world might be is represented in the pluriverse universal as a disjunct in the universal. Finally, we explain a main difficulty for the pluriverse universal as a metaphysical explanation of modality: it seems to require a previous domain of actual and merely possible objects.

Keywords: Modality, Metaphysics of Modality, Possible Worlds, Pluriverse, Universals.

$\mathbb{R A}$ Recientemente se ha propuesto una forma de generar mundos posibles ersatz mediante una única "gran" oración que representaría de una sola vez todos los hechos modales. Esta oración que expresa la totalidad de los hechos modales ha sido denominada "oración de pluriverso". La ventaja que tendría esta oración única por contraste con la forma usual en que las teorías modales lingüísticas han representado los hechos modales es que permitiría expresar hechos modales que conciernen a individuos posibles indiscernibles. En este trabajo se pretende explorar la viabilidad 
de utilizar una construcción teórica semejante para dar cuenta de individuos posibles indiscernibles, pero ahora en una concepción de los mundos posibles como universales estructurales. En la primera parte, por lo tanto, se van a explicar los lineamientos centrales y las motivaciones de la oración de pluriverso introducida por Theodor Sider para refinar una concepción lingüística de la modalidad. En la segunda parte se indicará qué forma debería tener el pluriverso cuando la idea general se incorpora a una concepción en la que los mundos posibles son entendidos como universales estructurales máximos. Finalmente, se considerarán algunas dificultades para el universal de pluriverso concebido de este modo.

\section{1. ¿Un pluriverso o muchos mundos posibles?}

La apelación a mundos posibles se ha visto como un recurso fructífero para la aclaración de las nociones modales y de una serie de otras nociones metafísicas. ¿Cómo deben ser entendidos estos "mundos posibles", sin embargo? David Lewis ha propuesto tomarlos sencillamente como "universos aislados", cosas de la misma naturaleza que el mundo actual ${ }^{1}$. Para muchos, no obstante, los costos de asumir esta ontología extravagante son demasiados, por lo que hablar de "mundos posibles" solo debería ser aceptable si es que estos pueden ser interpretados como algún tipo de "construcción" abstracta preservando al mundo actual como el único mundo concreto. Se han propuesto varias formas de realizar estas "construcciones". La postulación de una oración de pluriverso es un refinamiento de las teorías lingüísticas, en las que los mundos posibles son -en principio- conjuntos máximamente consistentes de oraciones de algún lenguaje que entregan una descripción o "relato" completo de cómo podría ser el mundo, dadas las capacidades expresivas del lenguaje en el que el relato se formula. Esto es, sea $\mathrm{S}$ el conjunto máximamente consistente que se pretende construir. Dado un conjunto $\left\{\alpha_{1}, \alpha_{2}, \ldots, \alpha_{n}\right\}$ de oraciones bien formuladas de un lenguaje L, sucede que para cada una de tales oraciones $\alpha_{i}$, o bien $\alpha_{i} \in \mathrm{S}$, o bien $\neg \alpha_{i} \in \mathrm{S}$. La teoría modal lingüística es atractiva porque parece exigir compromisos ontológicos no muy costosos. Tiene, sin embargo, el problema fundamental de que restringe el espacio modal de lo posible a las capacidades de expresión contingentes

1 En la concepción de Lewis el "mundo actual" es sencillamente un término indexical que designa el mundo en el que habita el hablante, del mismo modo que "aquí" designa el lugar en el que se encuentra el hablante, pero sin que esto confiera privilegios ontológicos para el lugar en el que alguien se encuentre por sobre otros lugares. Los mundos posibles lewisianos son cosas maximales, esto es, sumas mereológicas máximas de todos los objetos que satisfacen cierta condición: encontrarse todos ellos conectados espacio-temporalmente. Esto impide de entrada relaciones causales entre estos "universos paralelos" y también excluye de entrada individuos existentes en más de un mundo posible. Para presentaciones generales de la ontología modal de Lewis, cf. Lewis 1986a, pp. 1-96; Divers 2002, pp. 41-165; Melia 2003, pp. 99-121. 
que pueda tener el lenguaje en el que se expresan los "relatos" completos. Es obvio que un lenguaje natural de aquellos que empleamos ordinariamente no posee nombres para cada entidad existente. Posiblemente ni siquiera es posible que tengamos tal lenguaje como un lenguaje "utilizable", dado el carácter finito de nuestras capacidades de procesamiento cognitivo. Dadas estas dificultades se ha propuesto pensar en un lenguaje idealizado en el siguiente sentido: se tomará cada entidad del mundo como un nombre de sí misma. Este lenguaje ha sido denominado "lagadoniano" y permite de entrada resolver cualquier problema de capacidad expresiva que pudiese darse en un lenguaje natural ${ }^{2}$. No hay nada que no pueda expresarse en este lenguaje, si es que concierne a entidades actuales, pues -por definición-habrá en el lenguaje lagadoniano un nombre para cada objeto y para cada propiedad.

La teoría lingüística, sin embargo, ni aun con este fortalecimiento se encuentra libre de problemas en su capacidad expresiva. En principio, parece intuitivamente obvio que podría haber entidades que no existen actualmente. ¿Cómo se podría hablar sobre tales entidades en una concepción lingüística? No poseemos ningún nombre para tales entidades, pues los nombres propios que poseemos en el lenguaje lagadoniano son abundantes, pero solo designan entidades actuales. Tal vez se podrían expresar las posibilidades relativas a entidades posibles no actuales descriptivamente. No podemos, en efecto, nombrar directamente a un objeto posible no actual, pero sí podríamos hablar de algo que satisface un predicado $\mathrm{P}$. El problema que tiene este procedimiento es que parece también intuitivamente obvio que podría haber varios objetos que satisficiesen exactamente las mismas descripciones ${ }^{3}$. Podríamos pensar, por ejemplo, en un mundo con dos esferas perfectas de acero a cierta distancia d entre sí y que poseen exactamente las mismas propiedades intrínsecas y las mismas relaciones externas entre sí. Cada descripción que sea atribuible a una de estas esferas es también atribuible a la otra, por lo que no hay respectos descriptivos por los que puedan ser diferenciadas entre sí. Este ejemplo puede ser generalizado para n esferas perfectas a cierta distancia $d$ entre ellas, eventualmente infinitas esferas. Tampoco es necesario pensar en esferas perfectas de acero, lo mismo valdría si suponemos un mundo en el que se produce una secuencia compleja de eventos en el tiempo y en el espacio, como la historia de nuestro mundo, replicada exactamente en otra región del espacio o en

2 El recurso a un lenguaje lagadoniano fue introducido por Lewis (cf. Lewis 1986a, pp. 145-150), pero no para defender una forma de teoría lingüística, sino para argumentar que ni aun así la teoría lingüística sería aceptable. Es obvio -como apunta un evaluador anónimo-que un "lenguaje" semejante no podría ser aprendido por seres racionales como nosotros ni utilizable por seres racionales como nosotros, lo que hace muy dudoso que pueda ser calificado legítimamente como tal.

3 Para esta objeción, cf. Lewis 1986a, pp. 157-165. Lewis se concentra en el problema de representación de propiedades no ejemplificadas en el mundo actual, llamadas ordinariamente propiedades "extranjeras" (alien). El problema en relación con individuos no es tan urgente para él, dados sus compromisos metafísicos con contrapartidas. Aquí se centrará la discusión en individuos posibles indiscernibles, pero lo mismo podría luego aplicarse, mutatis mutandis, para propiedades extranjeras. 
muchas otras regiones del espacio ${ }^{4}$. Si los objetos posibles no actuales sólo pueden venir dados descriptivamente, ¿cómo se puede decir que hay dos objetos diferentes que satisfacen exactamente la misma descripción? Es más, así como parece intuitivamente obvio que existan dos objetos diferentes en un mundo posible $w_{1}$ que satisfacen exactamente las mismas descripciones, parece también intuitivamente obvio que podría haber un mundo posible $w_{2}\left(w_{2} \neq w_{1}\right)$ en que solo una de las esferas exista y la otra no, como también que podría haber un tercer mundo posible $w_{3}\left(w_{3} \neq w_{2}, w_{3} \neq w_{1}\right)$ en que la situación se invierta y solo exista la segunda esfera y la primera no.

El problema es que si se sostiene que los hechos modales están constituidos por lo que aparece representado en "relatos" completos (los mundos posibles del actualista en la concepción lingüística) y en tales relatos no pueden ser expresadas situaciones en las que varios objetos sean indiscernibles entre sí, entonces deberemos concluir que esas situaciones no son realmente posibles. Si tales casos de indiscernibilidad son posibles, entonces se debería rechazar la teoría lingüística. Este es el punto en que se introduce la idea de una oración de pluriverso. Theodor Sider ha sostenido que una forma de resolver estos problemas de capacidad expresiva es sustituir la pluralidad de conjuntos de oraciones máximamente consistentes por una sola gran oración que ha de expresar todos los hechos modales (cf. Sider 2002). El problema surge, sostiene Sider, porque las descripciones de lo que sucede en cada mundo posible solo dan cuenta de lo que acaece en ese mundo y no pueden servir para poner en relación lo que sucede ahí con lo que sucede en otros mundos diferentes. Así, por ejemplo, se tendrían descripciones como las siguientes:

$$
\begin{aligned}
& \exists w_{1} \text { En } w_{1}: \exists x_{1} \exists x_{2} \ldots \exists x_{\mathrm{n}} \exists P_{1} \exists P_{2} \ldots \exists P_{\mathrm{n}}\left(P_{1} x_{1} \wedge P_{2} x_{2} \wedge \ldots \wedge P_{\mathrm{n}} x_{\mathrm{n}}\right) \\
& \exists w_{2} \text { En } w_{2}: \exists x_{1} \exists x_{2} \ldots \exists x_{\mathrm{n}} \exists P_{1} \exists P_{2} \ldots \exists P_{\mathrm{n}}\left(P_{1} x_{2} \wedge P_{2} x_{1} \wedge \ldots \wedge P_{\mathrm{n}} x_{\mathrm{n}}\right) \\
& \ldots \\
& \exists w_{\mathrm{n}} \text { En } w_{\mathrm{n}}: \exists x_{1} \exists x_{2} \ldots \exists x_{\mathrm{n}} \exists P_{1} \exists P_{2} \ldots \exists P_{\mathrm{n}}\left(P_{1} x_{\mathrm{n}} \wedge P_{2} x_{\mathrm{n}-1} \wedge \ldots \wedge P_{\mathrm{n}} x_{1}\right)
\end{aligned}
$$

En estas descripciones, $w_{1}, w_{2}, \ldots, w_{\mathrm{n}}$ son mundos posibles, $x_{1}, x_{2}, \ldots, x_{\mathrm{n}}$ son objetos y $P_{1}, P_{2}, \ldots, P_{\mathrm{n}}$ son propiedades. Debe suponerse, por supuesto, que estas descripciones están dadas en un lenguaje infinitario. Considérese la diferencia entre los mundos posibles $w_{1}$ y $w_{2}$. Todo lo que dice cada una de las descripciones regidas por el cuantificador existencial inicial $(\exists w: .$.$) es que algo cae bajo P_{1}$ y algo cae bajo

$4 \quad$ La discusión de estos casos de individuos indiscernibles provienen de Black (1999). Una presentación de las consecuencias de estos casos de indiscernibilidad en metafísica modal en Adams (1979). He desarrollado con detalle por qué considero que el principio de identidad de los indiscernibles debe ser rechazado en Alvarado (2007), por lo que no entraré a discutir esta cuestión aquí y supondré simplemente que el principio es falso. 
$P_{2}$. Las variables utilizadas en tales descripciones para indicar cuál es este "algo" han sido intercambiadas en cada caso. En $w_{1}$ es $x_{1}$ el que cae bajo $P_{1}$ y es $x_{2}$ el que cae bajo $P_{2}$, mientras que en $w_{2}$ es $x_{2}$ el que cae bajo $P_{1}$ y es $x_{1}$ el que cae bajo $P_{2}$. Esto no permite expresar sin embargo que es el mismo objeto que en $w_{1}$ cae bajo $P_{1}$ el que en $w_{2}$ cae bajo $P_{2}$. La diferencia entre $w_{1}$ y $w_{2}$ es simplemente una variación alfabética de variables 5 . Todo lo que se dice en cada una de estas descripciones "completas" es que las propiedades $P_{1}, P_{2}, \ldots, P_{\mathrm{n}}$ están ejemplificadas, pero nada se dice sobre qué es lo que ejemplifican los objetos que aparecen ahí en otros mundos posibles. La forma de resolver esta dificultad sugerida por Sider es dar a los cuantificadores que rigen sobre mundos posibles, objetos y propiedades, el máximo alcance, generando una oración de una forma parecida a ésta:

(OP) $\exists w_{1} \exists w_{2} \ldots \exists w_{\mathrm{n}} \exists x_{1} \exists x_{2} \ldots \exists x_{\mathrm{n}} \exists P_{1} \exists P_{2} \ldots \exists P_{\mathrm{n}}:\left[\right.$ En $\left.w_{1}:\left(P_{1} x_{1} \wedge P_{2} x_{2} \wedge \ldots \wedge P_{\mathrm{n}} x_{\mathrm{n}}\right)\right] \wedge$ $\left[\right.$ En $\left.w_{2}:\left(P_{1} x_{2} \wedge P_{2} x_{1} \wedge \ldots \wedge P_{\mathrm{n}} x_{\mathrm{n}}\right)\right] \wedge \ldots \wedge\left[\operatorname{En} w_{\mathrm{n}}:\left(P_{1} x_{\mathrm{n}} \wedge P_{2} x_{\mathrm{n}-1} \wedge \ldots \wedge P_{\mathrm{n}} x_{1}\right)\right]$

Nuevamente, debe suponerse aquí que se trata de una oración infinitaria. Aquí, sin embargo, sí se expresa que los roles que cumplen $x_{1}$ y $x_{2}$ están invertidos en los mundos posibles $w_{1}$ y $w_{2}$, pues en $w_{1} x_{1}$ cae bajo $P_{1}$ y $x_{2}$ cae bajo $P_{2}$, mientras en $w_{2}$ es $x_{2}$ el que cae bajo $P_{1}$ y $x_{1}$ cae bajo $P_{2}$. Como estas variables están ligadas por los mismos cuantificadores, lo que dice la oración de pluriverso es que es el mismo objeto que en $w_{1}$ cae bajo $P_{1}$ el que en $w_{2}$ cae bajo $P_{2}$, de la misma manera que uno podría decir que:

$\exists x \exists y[($ En Francia: $((x$ es famoso $) \wedge(y$ es desconocido $))) \wedge($ En Inglaterra: $((x$ es desconocido $) \wedge(y$ es famoso $)))]$

Está claro en esta oración que el mismo objeto que es famoso en Francia es desconocido en Inglaterra y el mismo objeto que es famoso en Inglaterra es desconocido en Francia. Los cuantificadores que rigen la oración completa permiten "rastrear" lo que sucede con los objetos respectivos en diferentes mundos posibles (o en diferentes países, si se quiere).

No es necesario entrar aquí a explicar los detalles de la forma en que Sider formula la oración de pluriverso ni las restricciones que impone para su adecuación ${ }^{6}$. Hay, por supuesto, graves problemas con la propuesta de Sider -como también con

5 En efecto, en términos generales, la oración $\left[\exists x_{1} \mathrm{~F} x_{2}\right]$ y la oración $\left[\exists x_{2} \mathrm{~F} x_{2}\right]$ son la misma oración, dicen simplemente que algo cae bajo $F$. El que la expresión utilizada para señalar la variable sea diferente no hace ninguna diferencia. Toda la relevancia que tienen esas expresiones es clarificar exactamente qué variables está ligando un cuantificador determinado para diferenciarlas de las variables ligadas por otros cuantificadores y de las variables libres.

6 Cf. Sider 2002, pp. 290-307. Sider primero estipula "modelos modales realistas" en los que debe ser interpretada la oración de pluriverso. Luego muestra cómo la oración de pluriverso es adecuada para expresar los hechos de esos modelos modales realistas. 
cualquier otra teoría lingüística de la modalidad. En efecto, considérese que la oración de pluriverso (OP) parte diciendo que hay una serie de entidades: mundos, objetos y propiedades. Muchos de esos objetos no son actuales, como también muchas de las propiedades sobre las que se cuantifica ahí no están actualizadas. ¿Qué son esas entidades sobre las que se está cuantificando? Se espera de una teoría metafísica de la modalidad aceptable que explique qué son las entidades y los estados de cosas posibles que parecemos presuponer en nuestros razonamientos y nuestras intuiciones modales, pero aquí, lejos de ofrecérsenos una explicación de tales entidades, sencillamente se las da por supuestas. Debemos suponer que, por ejemplo, es posible que exista un gato posible no actual que baila y que podría no bailar, sencillamente porque una oración dice que hay un gato en un mundo posible que baila y que ese mismo gato en otro mundo posible no baila. ¿Por qué debemos aceptar que lo que dice una oración sobre lo que podría o no podría suceder es verdadera? ¿Por qué debemos otorgarle crédito precisamente a esa oración y no a cualquiera otra? Es obvio, en efecto, que hay muchas oraciones de pluriverso. Para el caso de las oraciones comunes con las que hablamos del mundo actual, hay hechos que discriminan entre las oraciones verdaderas y las oraciones falsas, aunque muchas veces nosotros no sepamos cuáles son verdaderas y cuáles son falsas. En este caso, sin embargo, ¿qué es lo que permite discriminar entre oraciones de pluriverso verdaderas de oraciones falsas? Si hay hechos modales que efectúan este trabajo, entonces la teoría lingüística es ociosa como explicación metafísica, si no los hay, entonces la teoría lingüística debe tomarse como una forma de anti-realismo modal de un tipo bien extremo, en la que lo posible depende de qué oración "decidamos" aceptar como determinando los hechos modales. Pareciera, por lo tanto, que una concepción realista de la modalidad debería buscar una explicación alternativa.

\section{Universales estructurales máximos}

Lo que se pretende aquí es explorar una idea análoga para la concepción de los mundos posibles como universales estructurales máximos. Esto exige algunas explicaciones previas sobre qué es lo que debe entenderse aquí por un universal de este estilo. La idea general de interpretar los mundos posibles como propiedades proviene inicialmente de Stalnaker (2003), pero las formulaciones más cercanas a lo que es propuesto aquí se encuentran en Forrest (1986a) y Bigelow y Pargetter (1990, pp. 93213). David Lewis sostiene que es una creencia generalmente aceptada que las cosas podrían ser diferentes de cómo son y, luego, que hay formas en que podrían ser las cosas que no son la forma en que las cosas son actualmente. En esta oración aparece una cuantificación existencial que puede tomarse tal como aparece, diciendo que se dan entidades de cierto tipo: "formas en que podrían ser las cosas". Todos creemos que hay posibilidades no realizadas y esto significa que todos creemos que hay formas en que podrían ser las cosas. Pues bien, los mundos posibles son simplemente esas formas en que podrían ser las cosas (cf. Lewis 1973, p. 84). Por supuesto, Lewis quiere tomar esto como una razón para aceptar la postulación de mundos posibles tal como 
él los entiende, pero no es necesario que esto sea así. La concepción de los mundos posibles como propiedades entiende estas entidades como "formas", esto es, propiedades que podría poseer el mundo, aunque de hecho no las posea. Han de tratarse de propiedades que especifiquen completamente cómo estaría constituido el mundo, si es que las ejemplificase. Una y solo una de estas propiedades se encuentra ejemplificada por el mundo actual ${ }^{7}$.

Hay muchas formas en que podría ser comprendida una propiedad. Por supuesto, ningún tipo de nominalismo podría servir para construir mundos posibles como propiedades, pues en estas teorías, las propiedades son determinadas por otros hechos ontológicos de un carácter más básico (por ejemplo, la existencia de predicados en un lenguaje, la existencia de conceptos en la mente, la existencia de clases, la existencia de semejanzas primitivas entre objetos, etcétera). Tampoco sería de mucha utilidad emplear tropos para "construir" los mundos posibles, pues se hace necesario dar aquí una explicación independiente de qué sería un "tropo posible no actual", mediante el que deberían construirse mundos posibles no actuales. Es difícil explicar qué es un tropo posible si no es por apelación a un possibile que lo ejemplifica, lo que torna la concepción irrelevante como teoría metafísica de la modalidad. La teoría modal basada en propiedades parece requerir universales para "construir" mundos posibles y universales trascendentes, esto es, universales no ejemplificados. En efecto, en esta concepción, un mundo posible no actual es un universal muy complejo que no está ejemplificado. Si solo hubiese universales ejemplificados, entonces solo podría aceptarse el universal que de hecho viene dado en el mundo actual. Como no habría más que un único mundo posible, se produciría un colapso modal en que $[p \rightarrow \square p]$.

¿Cómo es que un universal puede especificar "completamente" cómo es que podría estar constituido el mundo? La forma de hacerlo es mediante la especificación completa de cómo podría estar constituida cada una de las partes del mundo y, luego, mediante la indicación de las relaciones externas entre estas partes. Esto es exactamente lo que hace un universal estructural como, por ejemplo, ser una molécula de agua. Algo es agua solo si posee dos átomos de hidrógeno y un átomo de oxígeno relacionados entre sí mediante enlaces determinados. Así, el universal de ser una molécula de agua se atribuye a algo que posee tres partes, una de las cuales es un átomo de oxígeno y dos de las cuales son átomos de hidrógeno y entre las que se dan ciertos enlaces ${ }^{8}$. Un universal que sea capaz de "codificar" cómo sería el mundo debería tener cláusulas que especifican cómo serían sus partes (y luego, se pueden

7 Si se quiere, en la concepción modal basada en universales la expresión "el mundo actual" es ambigua, pues puede denotar el universal estructural máximo que de hecho se encuentra ejemplificado, o bien puede denotar lo que ejemplifica ese universal, esto es, lo que ordinariamente llamamos "el mundo".

$8 \quad$ Una explicación general de los universales estructurales en Armstrong (1997, pp. 32-38). En toda esta presentación se está asumiendo, naturalmente, una concepción realista de las propiedades como ítems objetivos de la realidad, que son el objeto básico de indagación de nuestras mejores teorías científicas. 
enunciar relaciones externas entre estas partes). Cada una de las partes en cuestión será, a su vez, especificada, porque se le atribuirá o se le negará cada uno de los universales $U_{1}, U_{2}, \ldots, U_{\mathrm{n}}$. Naturalmente, la especificación de cómo sería una parte de un mundo posible puede hacerse, a su vez, mediante la especificación de cómo serían las partes de esa parte. Las partes de una parte pueden luego tener el mismo tratamiento, etc. Las cláusulas de un universal estructural máximo, entonces, tendrían esta forma:

(UE) $\lambda x \exists y\left[(y<x) \wedge U_{1} y \wedge U_{2} y \wedge \ldots \wedge U_{\mathrm{n}} y\right]$

Aquí, '<' es el conectivo mereológico de "ser una parte (propia) de”. La cláusula, entonces, especifica la propiedad de ser un $x$ tal que $(\lambda x)$ : hay una parte propia $y$ de $x$ que instancia los universales $U_{1}, U_{2}, \ldots, U_{\mathrm{n}}$. Cada uno de estos universales, a su vez, puede tener la misma forma que (UE).

David Lewis ha expresado dudas de que la noción de un universal estructural sea inteligible (cf. Lewis 1986b). Los motivos fundamentales de desconfianza de Lewis tienen que ver con la dificultad de comprender cómo es que un universal estaría "compuesto" de otros. Por un lado, no parece que pueda verse a un universal estructural como una "construcción" conjuntista a partir de otros universales. Una construcción conjuntista exige partir desde un dominio dado de "simples" que aquí podrían no darse de ningún modo, pues puede suceder que todos los universales sean estructurales. Por otro lado, tampoco puede verse a un universal estructural como una suma mereológica de sus partes, pues en mereología, si dos objetos tienen exactamente las mismas partes son el mismo objeto. Aquí, en cambio, debería poder explicarse cómo es que pueden darse universales estructurales diferentes que poseen las mismas partes, esto es, los mismos universales. El metano, por ejemplo, está compuesto por átomos de carbono e hidrógeno, pero el butano también está compuesto por carbono e hidrógeno, pero son compuestos químicos completamente diferentes. No es posible entrar aquí en los detalles de esta discusión y de cómo puede ser comprendido un universal estructural (cf. Armstrong 1986, Forrest 1986b, Lewis 1986c, Bigelow 1986, Bigelow y Pargetter 1990, pp. 82-92). Sencillamente se va a suponer aquí que un universal estructural es aquel universal que se puede atribuir con verdad a aquellas entidades que satisfacen un predicado de la forma de (UE). Esto es neutral en relación con las diferentes teorías que puedan luego ser propuestas para explicar la naturaleza íntima de los universales estructurales.

Es obvio, sin embargo, que una concepción de los mundos posibles basada en universales tendrá las mismas dificultades que la teoría lingüística para representar posibilidades que envuelven entidades indiscernibles meramente posibles. En esta concepción, en efecto, no hay literalmente "objetos posibles no actuales". Lo único que hay son universales que podrían ser ejemplificados. Tal vez uno se sentiría tentado a sustituir, entonces, los objetos meramente posibles por esencias individuales que especificasen, mediante universales, las condiciones necesarias y suficientes para que 
exista un individuo particular en un mundo posible ${ }^{9}$. Los casos que se han indicado arriba de individuos indiscernibles entre sí, sin embargo, muestran de manera fehaciente que no existen semejantes esencias individuales ${ }^{10}$. Nuestra intuición es que podrían haber, por ejemplo, dos esferas perfectas de acero a cierta distancia d entre sí que ejemplifican exactamente los mismos universales y, luego, que podría existir la primera y no la segunda, o que podría existir la segunda y no la primera. ¿Cómo se representa esto mediante universales estructurales?

Hay que hacer notar que un universal estructural podría representar el hecho de que un mundo que lo ejemplificase tuviese dos esferas perfectas de acero a cierta distancia d entre sí. Sea el conjunto de universales que determina cada una de las esferas $\left\{U_{1}, U_{2}, \ldots, U_{\mathrm{n}}\right\}$, sea la relación de "encontrarse a una distancia $d$ " $R$. Es perfectamente inteligible un universal de esta estructura:

$$
\begin{aligned}
& \lambda x \exists y \exists z\left[(y<x) \wedge(z<x) \wedge\left(U_{1} y \wedge U_{2} y \wedge \ldots \wedge U_{\mathrm{n}} y\right) \wedge\left(U_{1} z \wedge U_{2} z \wedge \ldots \wedge U_{\mathrm{n}} z\right)\right. \\
& \wedge R y z \wedge(y \neq z)]
\end{aligned}
$$

Esto es, la propiedad de poseer una parte $y$ que ejemplifica los universales $U_{1}$, $U_{2}, \ldots, U_{\mathrm{n}}$, de poseer una parte $z$ que ejemplifica exactamente los mismos universales $U_{1}, U_{2}, \ldots, U_{\mathrm{n}}$, y en que $y$ es diferente de $z$ y se encuentran a cierta distancia $d$ entre sí. Todo lo que se requiere para construir este universal es admitir que la cuantificación existencial corresponde a un universal ("ejemplificarse en algún objeto"), el operador mereológico de "ser una parte propia de" es un universal, la identidad (y su complemento) son también universales y que, finalmente, la conjunción es un mecanismo admisible para construir universales a partir de otros universales ${ }^{11}$. Ninguno de estos supuestos resulta terriblemente inverosímil. El problema que subsiste aquí, sin embargo, es la representación de hechos de identidad y de diferencia entre mundos posibles. En efecto, se puede representar que un mundo posible $w_{1}$ es tal que en él habría dos esferas perfectas de acero, se puede luego representar que en otro mundo posible $w_{2}$ hay solo una esfera de acero, pero no hay cómo representar que la única esfera de acero de $w_{2}$ es o no idéntica a una de las dos esferas de acero de $w_{1}$. Esto se podría hacer si hubiese un universal que codificase exactamente en qué consiste ser esa esfera de acero en particular, pero no poseemos tal cosa.

$9 \quad$ Tal como lo hace, por ejemplo, Plantinga (1974, pp. 70-77).

10 A menos que uno introduzca en la esencia un elemento de haecceitas, tal como decir que un individuo $b$ ha de tener entre sus propiedades esenciales la de ser una instancia de $[\lambda x$ $(x=b)]$. Esto depende de la existencia previa de $b$, sin embargo, y aquí se pretende eliminar objetos posibles no actuales de la ontología. Plantinga propone una forma alternativa de especificar esencias individuales que también presupone la existencia de individuos posibles no actuales y, es más, de mundos posibles con todos sus hechos modales establecidos (cf. Plantinga 1974, pp. 70-77).

11 Esto es algo que el mismo Lewis admite; cf. Lewis 1986b, p. 41, nota 21. 


\section{El universal de pluriverso}

La dificultad que plantea la representación de objetos posibles indiscernibles podría, tal vez, ser tratada mediante una estrategia análoga a la empleada para resolver las limitaciones expresivas de los mundos posibles lingüísticos. En vez de pensar en un universal estructural máximo por cada mundo posible se debería pensar en un solo universal de pluriverso que "codificase" de alguna manera la totalidad de los hechos modales. En este universal estarían inscritas las relaciones de identidad y diferencia entre distintos mundos posibles mediante cuantificadores con el máximo alcance. Luego, el universal de pluriverso contendría cláusulas disyuntivas con especificaciones completas de cómo podría ser el mundo, en los términos ya explicados, esto es, mediante cláusulas que indicarían cómo sería cada una de las partes del mundo y qué relaciones externas tendría cada una de esas partes con las restantes partes de ese mismo mundo. La estructura del universal de pluriverso, entonces, sería la siguiente:

$$
\begin{aligned}
& \lambda y \exists x_{1} \exists x_{2} \ldots \exists x_{\mathrm{n}}\left[\left(( ( x _ { 1 } < y ) \wedge U _ { 1 } x _ { 1 } \wedge U _ { 2 } x _ { 1 } \wedge \ldots \wedge U _ { \mathrm { n } } x _ { 1 } ) \wedge \left(\left(x_{2}<y\right) \wedge U_{2} x_{2} \wedge U_{3} x_{2}\right.\right.\right. \\
& \left.\left.\wedge \ldots \wedge U_{\mathrm{n}+1} x_{2}\right) \wedge \ldots\left(\left(x_{\mathrm{n}}<y\right) \wedge U_{\mathrm{n}} x_{\mathrm{n}} \wedge U_{\mathrm{n}+1} x_{\mathrm{n}} \wedge \ldots \wedge U_{\mathrm{n}+\mathrm{m}} x_{\mathrm{n}}\right)\right) \vee\left(\left(\left(x_{1}<y\right) \wedge U_{2} x_{1}\right.\right. \\
& \left.\wedge U_{3} x_{1} \wedge \ldots \wedge U_{\mathrm{n}+1} x_{1}\right) \wedge\left(\left(x_{2}<y\right) \wedge U_{3} x_{2} \wedge U_{4} x_{2} \wedge \ldots \wedge U_{\mathrm{n}+2} x_{2}\right) \wedge \ldots\left(\left(x_{\mathrm{n}}<y\right) \wedge\right. \\
& \left.U_{\mathrm{n}} x_{\mathrm{n}} \wedge U_{\mathrm{n}+1} x_{\mathrm{n}} \wedge \ldots \wedge U_{\mathrm{m}} x_{\mathrm{n}}\right) \vee \ldots \ldots \vee\left(\left(x_{1}<y\right) \wedge U_{\mathrm{m}} x_{1} \wedge U_{\mathrm{m}+1} x_{1} \wedge \ldots \wedge U_{\mathrm{m}+\mathrm{s}} x_{1}\right) \wedge \\
& \left(\left(x_{2}<y\right) \wedge U_{\mathrm{m}+1} x_{2} \wedge U_{\mathrm{m}+2} x_{2} \wedge \ldots \wedge U_{\mathrm{m}+\mathrm{q}} x_{2}\right) \wedge \ldots\left(\left(x_{\mathrm{n}}<y\right) \wedge U_{\mathrm{q}} x_{\mathrm{n}} \wedge U_{\mathrm{q}+1} x_{\mathrm{n}} \wedge \ldots \wedge\right. \\
& \left.\left.\left.U_{\mathrm{q}+\mathrm{s}} x_{\mathrm{n}}\right)\right)\right]
\end{aligned}
$$

Esto es, la propiedad de ser un $y$ que posee como partes objetos $x_{1}, x_{2}, \ldots, x_{\mathrm{n}} \mathrm{y}$ universales $U_{1}, U_{2}, \ldots, U_{\mathrm{n}} \mathrm{y}$, o bien se da que $U_{1} x_{1}$ y $U_{2} x_{2}$ y etcétera hasta conseguir una especificación completa de cómo sería el mundo, o bien es el caso que se daría otra especificación completa de cómo sería el mundo, o bien se daría otra diferente y etcétera, hasta agotar todas las formas en que podría ser el mundo. Recuérdese que se especifica cómo sería el mundo en cada una de las cláusulas disyuntivas de (UP) dado como serían sus partes, por lo que la descripción de las partes tiene la forma de (UE). La descripción de esas partes puede darse nuevamente en términos de sus respectivas partes, etc. Considérese, entonces, el problema indicado arriba de dos esferas perfectas de acero a cierta distancia $d$ entre sí, sean $a$ y $b$, en un mundo posible $w_{1}$, mientras en $w_{2}\left(w_{2} \neq w_{1}\right)$ solo existe $a$ y no existe $b$, y en $w_{3}\left(w_{3} \neq w_{2}\right.$ y $\left.w_{3} \neq w_{1}\right)$ solo existe $b$ y no existe $a$. Se ha explicado cómo es que un universal podría expresar el hecho de que podrían darse dos esferas perfectas de acero en un mundo posible, pero las identidades y diferencias entre objetos entre distintos mundos posibles parecían quedar fuera de las capacidades de representación de los universales estructurales. Pues bien, sean de nuevo los universales $U_{1}, U_{2}, \ldots, U_{\mathrm{n}}$ los que especifican completamente cómo sería cada esfera de acero. Sea $R$ la relación de encontrarse a cierta distancia $d$ entre sí. Se puede ahora construir el siguiente universal estructural:

$$
\begin{aligned}
& \lambda x \exists y \exists z\left[\left[(y<x) \wedge(z<x) \wedge\left(U_{1} y \wedge U_{2} y \wedge \ldots \wedge U_{\mathrm{n}} y\right) \wedge\left(U_{1} z \wedge U_{2} z \wedge \ldots \wedge U_{\mathrm{n}} z\right)\right.\right. \\
& \wedge R y z \wedge(y \neq z)] \vee\left[(y \leq x) \wedge\left(U_{1} y \wedge U_{2} y \wedge \ldots \wedge U_{\mathrm{n}} y\right) \wedge(\neg \exists v(v<x) \wedge(v \neq y))\right] \\
& \left.\vee\left[(z \leq x) \wedge\left(U_{1} z \wedge U_{2} z \wedge \ldots \wedge U_{\mathrm{n}} z\right) \wedge(\neg \exists v(v<x) \wedge(v \neq z))\right]\right]
\end{aligned}
$$


En este universal estructural se especifica la propiedad de ser tal que hay dos objetos y y $z$, tales que, o bien son dos esferas perfectas de acero a cierta distancia $d$ entre sí, o bien solo existe la esfera de acero $y$, o bien solo existe la esfera de acero $z$. Los cuantificadores al principio garantizan que es el mismo objeto $y$ aquel que aparece en la primera cláusula disyuntiva $\left[(y<x) \wedge(z<x) \wedge\left(U_{1} y \wedge U_{2} y \wedge \ldots \wedge U_{\mathrm{n}} y\right) \wedge\left(U_{1} z \wedge\right.\right.$ $\left.\left.U_{2} z \wedge \ldots \wedge U_{\mathrm{n}} z\right) \wedge R y z \wedge(y \neq z)\right]$ y en la segunda cláusula disyuntiva $\left[(y \leq x) \wedge\left(U_{1} y \wedge\right.\right.$ $\left.\left.U_{2} y \wedge \ldots \wedge U_{\mathrm{n}} y\right) \wedge(\neg \exists v(v<x) \wedge(v \neq y))\right]$. Del mismo modo, garantizan que es el mismo objeto $z$ el que aparece en la primera cláusula disyuntiva $\left[(y<x) \wedge(z<x) \wedge\left(U_{1} y\right.\right.$ $\left.\left.\wedge U_{2} y \wedge \ldots \wedge U_{\mathrm{n}} y\right) \wedge\left(U_{1} z \wedge U_{2} z \wedge \ldots \wedge U_{\mathrm{n}} z\right) \wedge R y z \wedge(y \neq z)\right]$ y en la tercera cláusula disyuntiva $\left[(z \leq x) \wedge\left(U_{1} z \wedge U_{2} z \wedge \ldots \wedge U_{\mathrm{n}} z\right) \wedge(\neg \exists v(v<x) \wedge(v \neq z))\right]$. Hay que pensar que en un universal de pluriverso las cláusulas disyuntivas entregan especificaciones completas de cómo sería un mundo posible en todos sus detalles y, en segundo lugar, hay cláusulas disyuntivas por cada forma en que podría ser un mundo. El universal estructural $(* *)$, por lo tanto, está lejos de ser un universal de pluriverso. De todos modos, permite comprender cómo es que funcionaría un universal de pluriverso para determinar hechos modales entre diferentes mundos posibles.

Una vez "construido" el universal de pluriverso, entonces, es posible replicar los análisis clásicos de nociones modales mediante mundos posibles, esta vez entendiendo los mundos posibles como cláusulas disyuntivas de (UP). Hay que hacer notar aquí que, naturalmente, (UP) está necesariamente ejemplificado, pues comoquiera que sea el mundo, una de las cláusulas disyuntivas expresa exactamente cómo es el mundo $^{12}$. Se tomarán las variables $D_{1}, D_{2}, \ldots D_{\mathrm{n}}$ como rigiendo sobre las cláusulas disyuntivas de (UP). Cuando se consideran estas cláusulas disyuntivas es esencial que sean tomadas bajo los cuantificadores que rigen sus variables. Esto es, deben tomarse como "conectadas" del modo que indican esos cuantificadores con las restantes formas en que podría ser el mundo, según esos cuantificadores han determinado. Si se tomasen las cláusulas disyuntivas aisladamente, toda la ganancia que ofrece (UP) se perdería. En lo que sigue, se utilizará la abreviación $[\alpha \leftrightarrow \beta \leftrightarrow \gamma]$ para expresar $[(\alpha \leftrightarrow$ $\beta) \wedge(\beta \leftrightarrow \gamma) \wedge(\alpha \leftrightarrow \gamma)]$. Los análisis clásicos se tomarían del siguiente modo:

( $\square) \quad(p$ es necesario $) \leftrightarrow(p$ es verdadero en todo mundo posible $w) \leftrightarrow$ (para todo $D$, si $D$ se ejemplificase $p$ sería verdadero)

$(\neg \oslash) \quad(p$ es imposible $) \leftrightarrow(p$ es falso en todo mundo posible $w) \leftrightarrow$ (para todo $D$, si $D$ se ejemplificase $p$ sería falso)

12 El universal de pluriverso es, en un sentido trivial, la esencia del mundo. En efecto, es necesario que, si el mundo existe, entonces el mundo ejemplifique (UP) porque trivialmente (UP) contiene todas las formas en que podría ser el mundo. Una forma usual de expresar una propiedad esencial $P$ de $x$ es decir que $[\square(x$ existe $\rightarrow P x)]$. En este caso, (UP) no solo sería una propiedad esencial del mundo, sino su esencia, porque si (UP) está ejemplificado, entonces el mundo existe. Esto es, es un universal cuya ejemplificación es a la vez necesaria y suficiente para que el mundo exista. 
( $p$ es posible) $\leftrightarrow(p$ es verdadero en algún mundo posible $w) \leftrightarrow$ (hay un $D$ tal que, si $D$ se ejemplificase $p$ es verdadero $)^{13}$

$(\diamond \& \neg \diamond) \quad(p$ es contingente $) \leftrightarrow\left(p\right.$ es verdadero en algún mundo posible $w_{1}$ y $p$ es falso en algún mundo posible $w_{2}$ ) $\leftrightarrow$ (hay un $D_{1}$ tal que, si $D_{1}$ se instanciase $p$ es verdadero y hay un $D_{2}$ tal que, si $D_{2}$ se instanciase $p$ es falso).

De una manera semejante deben ser analizados los enunciados de modalidad relativa, esto es, de modalidad según cierta restricción especial, como, por ejemplo, la que concierne a las leyes naturales (modalidad nomológica). El que en un mundo posible sean verdaderas ciertas leyes naturales debe tomarse sencillamente como el hecho de que ciertos universales se encuentran ejemplificados en ese mundo. Una modalidad relativa $R$ puede formularse al menos de dos modos alternativos. Un modo es restringir los cuantificadores en las cláusulas de la derecha a las disyunciones que

13 Se habrá notado que la formulación para las cláusulas de necesidad y de posibilidad son -al menos aparentemente- condicionales. Algo es necesario porque si se ejemplificase cualquier $D$, entonces eso sería el caso. Algo es posible porque si se ejemplificase algún $D$, entonces eso sería el caso. Esto pareciera estar en conflicto con la dualidad de los operadores modales de necesidad y posibilidad. En efecto, si $[\square p \leftrightarrow \neg \checkmark \neg p]$ y $\left[\square p=_{\mathrm{df}} \forall D(D\right.$ $\rightarrow p$ )] (aquí debe tomarse el definiens como la expresión formalizada de: para todo $D$, si $D$ se ejemplifica, entonces $p$; la implicación es material), entonces pareciera que [ $\diamond p \leftrightarrow \neg \forall D$ $(D \rightarrow \neg p)]$ y, luego, que $[\diamond p \leftrightarrow \exists D(D \wedge p)]$. Esto sería desastroso, pues se estaría diciendo que algo es posible si y solo si hay una cláusula disyuntiva de (UP) que está ejemplificada actualmente y p es actualmente el caso. Solo podrían ser posibles los estados de cosas actuales. Por esto, las implicaciones que aparecen en las formulaciones ( $\square)-(r e)$ deben entenderse como nociones modales, esto es, como condicionales contrafácticos ya sea del tipo could o del tipo might. En la explicación estándar de las condiciones de verdad de estos condicionales se los trata como duales, de manera que $[(p \square \rightarrow q) \leftrightarrow \neg(p \diamond \rightarrow \neg q)]$ y $[(p \diamond \rightarrow q) \leftrightarrow \neg(p \square \rightarrow \neg q)]$ (cf. Lewis 1973, pp. 21-24; Bennett 2003, pp. 189-193). Intuitivamente, el condicional contrafáctico could $[p \square \rightarrow q]$ es verdadero en el mundo posible $w_{\mathrm{i}}$ si y solo si en todos los mundos posibles más cercanos a $w_{\mathrm{i}}$ en que $p$ es verdadero, $q$ es también verdadero. Por otro lado, el condicional contrafáctico might $[p \diamond \rightarrow q]$ es verdadero en el mundo posible $w_{\mathrm{i}}$ si y solo si en algún mundo posible más cercano a $w_{\mathrm{i}} p$ y $q$ son ambos verdaderos. La cláusula de necesidad ( $\square$ ) debe entenderse, entonces, como $[\forall D(D \square \rightarrow p)$ ] y la cláusula de posibilidad $(\diamond)$ como $[\exists D(D \diamond \rightarrow p)]$. Puede parecer aquí extraño el hacer apelación a nociones modales que, en principio, deberían ser aclaradas precisamente por las entidades de las que trata la teoría modal basada en universales. Esto es simplemente un índice, sin embargo, de la imposibilidad de dar un análisis reductivo de las nociones modales en términos de nociones no-modales. No es la pretensión de la teoría modal basada en universales reducir la modalidad, sino simplemente dilucidarla. En cuanto al uso de condicionales contrafácticos para las formulaciones ( $\square)$-(re), estos pueden tomarse perfectamente como nociones primitivas que no presuponen el aparato semántico de los mundos posibles para su comprensión (cf. por ejemplo, Williamson 2007, pp. 293304). 
satisfacen las leyes naturales en cuestión (o lo que sea), sean $D^{\mathrm{R}}$. Así, las condiciones de verdad de enunciados modales relativos a $R$ se formularían del siguiente modo:

( $\square \mathrm{R}) \quad(p$ es $R$-necesario $) \leftrightarrow(p$ es verdadero en todo mundo posible $w$ en que $R) \leftrightarrow$ (para todo $D^{\mathrm{R}}$, si $D^{\mathrm{R}}$ se ejemplificase $p$ sería verdadero)

$(\diamond \mathrm{R}) \quad(p$ es $R$-posible $) \leftrightarrow(p$ es verdadero en algún mundo posible $w$ en que $R) \leftrightarrow$ (hay un $D^{\mathrm{R}}$ tal que, si $D^{\mathrm{R}}$ se ejemplificase $p$ es verdadero).

Otra forma alternativa de expresar las mismas condiciones de verdad es dejar los cuantificadores sobre cláusulas disyuntivas en la derecha irrestrictas, pero introducir la restricción $R$ al interior de la especificación de las condiciones de verdad, del siguiente modo:

( $\left.\square \mathrm{R}^{\prime}\right) \quad(p$ es $R$-necesario $) \leftrightarrow(p$ es verdadero en todo mundo posible $w$ en que $R) \leftrightarrow$ (para todo $D$, si $D$ se ejemplificase y $R$, entonces $p$ sería verdadero)

$\left(\diamond \mathrm{R}^{\prime}\right) \quad(p$ es $R$-posible $) \leftrightarrow(p$ es verdadero en algún mundo posible $w$ en que $R) \leftrightarrow$ (hay un $D$ tal que, si $D$ se ejemplificase y $R$, entonces $p$ es verdadero)

Tratándose de condicionales estrictos, esto es, condicionales de la forma $[\square(p$ $\rightarrow q$ )], el análisis tiene la siguiente forma:

$(\rightarrow) \quad(\square(p \rightarrow q)) \leftrightarrow($ en todos los mundos posibles $w$, o bien $\neg p$ o bien $q) \leftrightarrow$ (para todo $D$, si $D$ se ejemplificase, entonces o bien $\neg p$ o bien $q$ ).

El análisis de un condicional contrafáctico $[p \square \rightarrow q]$, en cambio, exige más precisiones. En el tratamiento de David Lewis, un condicional contrafáctico $[p \square \rightarrow$ $q$ ] es verdadero en un mundo posible $w_{\mathrm{i}}$ si y solo si, en todos los mundos posibles pertenecientes a la clase de mundos posibles más "cercanos" a $w_{\mathrm{i}}$, sea $S_{\mathrm{i}}$, es verdadera la implicación material $[p \rightarrow q]$ (cf. Lewis 1973, pp. 8-11). Qué sea aquí un mundo posible más "cercano" o más "lejano" depende de las semejanzas y diferencias entre mundos posibles. A su vez, cómo deban ser evaluadas tales semejanzas y diferencias es una cuestión relativamente vaga y dependiente del contexto de evaluación del condicional contrafáctico en cuestión. Nada de esto, sin embargo, está ajeno a lo que resulta admisible para el defensor de la concepción de mundos posibles como universales. Así como los mundos posibles de David Lewis pueden ser más o menos semejantes entre sí según diversos respectos, de la misma forma, los modos en que podría ser el mundo pueden ser más o menos semejantes entre sí (por ejemplo, dependiendo de qué universales sean ejemplificados en cada uno de ellos, aunque no solo por este motivo). No hay ningún inconveniente, entonces, en que respecto de una cláusula disyuntiva $D_{\mathrm{i}}$ se fije una clase de cláusulas disyuntivas "cercanas" $S_{\mathrm{i}}$ que han de ser relevantes en un contexto determinado para evaluar un condicional contrafáctico. Así, las condiciones de verdad quedan como sigue, pensando que se evalúa el condicional 
contrafáctico de la izquierda en un mundo posible determinado $w_{\mathrm{i}}$ (que puede ser, naturalmente, el mundo actual):

$(\square \rightarrow) \quad(p \square \rightarrow q) \leftrightarrow\left(\right.$ en todos los mundos posibles $w \in S_{\mathrm{i}}$, o bien $\neg p$ o bien $\left.q\right) \leftrightarrow$ (para todo $D \in S_{\mathrm{i}}$, si $D$ se ejemplificase, entonces o bien $\neg p$ o bien $q$ ).

Por último, en el caso de los enunciados modales de re en que se dice que un cierto individuo, sea $b$, podría ser $P$, las condiciones de verdad son obvias en este análisis:

(re) $\quad(b$ es posiblemente $P$ ) $\leftrightarrow$ (hay un mundo posible $w$ en que $b$ es $P$ ) $\leftrightarrow$ (hay un $D$ tal que, si $D$ se ejemplificase $P b$ es verdadero).

Los cuantificadores al principio de (UP) garantizan que es el mismo objeto $b$ aquel que en el mundo de evaluación es posiblemente $P$ y aquel objeto tal que si $D$ se ejemplificase, sería $P$.

\section{Dificultades para el universal de pluriverso}

La concepción modal basada en universales utilizando un universal de pluriverso como el que ha sido descrito, parece representar lo que intuitivamente creemos posible sobre possibilia indiscernibles. Es obvio, sin embargo, que hay dificultades o, al menos, "costos" que deben ser asumidos por esta teoría. Existe, en primer lugar, todo un conjunto de problemas que tienen que ver simplemente con los presupuestos metafísicos que exige esta "construcción". Los universales trascendentes no son precisamente populares (críticas en Armstrong (1978a, pp. 64-76; Armstrong 1989, pp. 75-82). Hay también dudas sobre la inteligibilidad de universales estructurales en general y, en particular, muchos filósofos que defienden una ontología de universales no aceptan, por ejemplo, la existencia de universales disyuntivos que resultan indispensables, tal como se ha visto, para (UP) (cf. Armstrong 1978b, pp. 19-29; Armstrong 1989, pp. 82-84). Una vez despejadas todas estas cuestiones, resta todavía por aclarar cómo podrían admitirse como universales integrando un universal estructural la existencia, la identidad y la relación mereológica de "ser parte de". No creo que ninguna de estas cuestiones sea un defecto fatal para la concepción modal basada en universales. Se trata de problemas que requieren atención detenida. Pero, nada parece obstar para pensar que tal atención detenida no sea suficiente para aclarar las dudas de muchos filósofos.

El tipo de dificultad que se planteará aquí tiene un carácter más específico que las dudas generales referidas a la ontología de universales. El universal de pluriverso incluye a la derecha del operador lambda una fila de cuantificadores objetuales que se destacan a continuación: 


$$
\begin{aligned}
& \lambda y \exists \boldsymbol{x}_{1} \exists \boldsymbol{x}_{2} \ldots \exists \boldsymbol{x}_{\mathrm{n}}\left[\left(( ( x _ { 1 } < y ) \wedge U _ { 1 } x _ { 1 } \wedge U _ { 2 } x _ { 1 } \wedge \ldots \wedge U _ { \mathrm { n } } x _ { 1 } ) \wedge \left(\left(x_{2}<y\right) \wedge U_{2} x_{2} \wedge\right.\right.\right. \\
& \left.\left.U_{3} x_{2} \wedge \ldots \wedge U_{\mathrm{n}+1} x_{2}\right) \wedge \ldots\left(\left(x_{\mathrm{n}}<y\right) \wedge U_{\mathrm{n}} x_{\mathrm{n}} \wedge U_{\mathrm{n}+1} x_{\mathrm{n}} \wedge \ldots \wedge U_{\mathrm{n}+\mathrm{m}} x_{\mathrm{n}}\right)\right) \vee\left(\left(\left(x_{1}<y\right)\right.\right. \\
& \left.\wedge U_{2} x_{1} \wedge U_{3} x_{1} \wedge \ldots \wedge U_{\mathrm{n}+1} x_{1}\right) \wedge\left(\left(x_{2}<y\right) \wedge U_{3} x_{2} \wedge U_{4} x_{2} \wedge \ldots \wedge U_{\mathrm{n}+2} x_{2}\right) \wedge \ldots\left(\left(x_{\mathrm{n}}\right.\right. \\
& \left.<y) \wedge U_{\mathrm{n}} x_{\mathrm{n}} \wedge U_{\mathrm{n}+1} x_{\mathrm{n}} \wedge \ldots \wedge U_{\mathrm{m}} x_{\mathrm{n}}\right) \wedge \ldots \vee\left(\left(x_{1}<y\right) \wedge U_{\mathrm{m}} x_{1} \wedge U_{\mathrm{m}+1} x_{1} \wedge \ldots \wedge\right. \\
& \left.U_{\mathrm{m}+\mathrm{s}} x_{1}\right) \wedge\left(\left(x_{2}<y\right) \wedge U_{\mathrm{m}+1} x_{2} \wedge U_{\mathrm{m}+2} x_{2} \wedge \ldots \wedge U_{\mathrm{m}+\mathrm{q}} x_{2}\right) \wedge \ldots\left(\left(x_{\mathrm{n}}<y\right) \wedge U_{\mathrm{q}} x_{\mathrm{n}} \wedge\right. \\
& \left.\left.U_{\mathrm{q}+1} x_{\mathrm{n}} \wedge \ldots \wedge U_{\mathrm{q}+\mathrm{s}} x_{\mathrm{n}}\right)\right]
\end{aligned}
$$

Pues bien, ¿sobre qué están rigiendo tales cuantificadores? Una motivación central de la concepción modal basada en universales es eliminar de nuestra ontología objetos meramente posibles. El reino de lo posible (no actual) está conformado aquí exclusivamente por propiedades no ejemplificadas, "formas" en que podrían ser las cosas. El problema es que en (UP) parece que de entrada este requerimiento se deja a un lado pues los cuantificadores estarían literalmente diciendo que hay objetos que ejemplifican tales y cuales propiedades. Algunos de esos objetos son actuales, otros son possibilia. Luego, la inteligibilidad de (UP) parece requerir previamente la existencia de possibilia y mal puede servir para explicar la naturaleza ontológica de tales entidades.

Una respuesta a esta dificultad sería sostener que el operador lambda al principio genera un contexto intencional y que, por lo tanto, no es necesario que literalmente existan las entidades sobre las que se cuantifica después, para que (UP) sea inteligible como propiedad. Por ejemplo, considérese un enunciado como el siguiente:

\section{$(* * *) \quad S$ cree que: $[\exists x F x]$}

Es obvio que este enunciado es inteligible aun cuando no existiese ningún $F$. Si el cuantificador regido por el operador doxástico " $S$ cree que..." fuese restringido y el dominio de cuantificación vacío, no habría nada sobre lo que se estaría cuantificando. Esto no impide que, incluso en estos casos, el enunciado (***) pueda ser verdadero si es que $S$ cree realmente que hay un $F$. De la misma manera, para que exista el universal (UP) no se requiere que existan entidades sobre las que rijan los cuantificadores a la derecha del operador lambda.

El problema que tiene esta respuesta es que, aun admitiendo que es inteligible un universal en que aparecen cuantificadores que no están rigiendo sobre nada, si es verdadero atribuir tal universal a algo, entonces las condiciones estipuladas en el universal deben cumplirse. Y, en efecto, (UP) no puede dejar de ser ejemplificado. Su ejemplificación es necesaria, pues comoquiera que esté constituido el mundo realmente, estará ejemplificando una de las cláusulas disyuntivas de (UP), por lo que (UP) se atribuirá con verdad del mundo. Si se atribuye con verdad del mundo, entonces, es el caso que:

$$
\begin{aligned}
& \exists x_{1} \exists x_{2} \ldots \exists x_{\mathrm{n}}\left[\left(( U _ { 1 } x _ { 1 } \wedge U _ { 2 } x _ { 1 } \wedge \ldots \wedge U _ { \mathrm { n } } x _ { 1 } ) \wedge \left(( U _ { 2 } x _ { 2 } \wedge U _ { 3 } x _ { 2 } \wedge \ldots \wedge U _ { \mathrm { n } + 1 } x _ { 2 } ) \wedge \ldots \left(\left(U_{\mathrm{n}} x_{\mathrm{n}} \wedge\right.\right.\right.\right.\right. \\
& \left.\left.U_{\mathrm{n}+1} x_{\mathrm{n}} \wedge \ldots \wedge U_{\mathrm{n}+\mathrm{m}} x_{\mathrm{n}}\right)\right) \vee\left(\left(U_{2} x_{1} \wedge U_{3} x_{1} \wedge \ldots \wedge U_{\mathrm{n}+1} x_{1}\right) \wedge\left(U_{3} x_{2} \wedge U_{4} x_{2} \wedge \ldots \wedge U_{\mathrm{n}+2} x_{2}\right) \wedge \ldots\right. \\
& \left.\left(U_{\mathrm{n}} x_{\mathrm{n}} \wedge U_{\mathrm{n}+1} x_{\mathrm{n}} \wedge \ldots \wedge U_{\mathrm{m}} x_{\mathrm{n}}\right)\right) \vee \ldots \vee\left(( U _ { \mathrm { m } } x _ { 1 } \wedge U _ { \mathrm { m } + 1 } x _ { 1 } \wedge \ldots \wedge U _ { \mathrm { m } + \mathrm { s } } x _ { 1 } ) \wedge \left(U_{\mathrm{m}+1} x_{2} \wedge U_{\mathrm{m}+2} x_{2}\right.\right. \\
& \left.\left.\wedge \ldots \wedge U_{\mathrm{m}+\mathrm{q}} x_{2}\right) \wedge \ldots\left(U_{\mathrm{q}} x_{\mathrm{n}} \wedge U_{\mathrm{q}+1} x_{\mathrm{n}} \wedge \ldots \wedge U_{\mathrm{q}+\mathrm{s}} x_{\mathrm{n}}\right)\right]
\end{aligned}
$$


Comoquiera que sea el mundo, hay objetos que ejemplifican tales y cuales universales, algunos de ellos son objetos actuales, otros son possibilia. Luego, sí se requieren possibilia para hacer inteligible (UP).

La situación teórica que parece resultar es incómoda. Si se quieren explicar las modalidades desde el punto de vista metafísico como dependientes de propiedades, entonces esto parece implicar que debemos renunciar a la inteligibilidad de posibilidades que envuelven a individuos no actuales (salvo en lo que pueda venir representado por universales). Si se introducen recursos suficientes para representar hechos sobre possibilia indiscernibles, entonces pareciera que debemos introducir en nuestra teoría elementos del todo ajenos al repertorio ontológico con el que se pretendía efectuar la explicación. Por supuesto, está siempre abierta la alternativa de asumir la teoría basada en universales y solo universales a cualquier costo. En este caso, habrá que declarar que realmente no son posibles los hechos putativos indicados más arriba sobre possibilia indiscernibles. Si nuestra intuición parecía indicarnos lo contrario, ello se debía simplemente a un error, tal como era un error la intuición según la cual la geometría del mundo físico era necesariamente euclidiana, o la intuición según la cual cualquier condición $\varphi$ serviría para determinar la clase de cosas que satisfacen $\varphi$. Esta posición puede ser complementada mediante el recurso de las contrapartidas (cf. Lewis 1983) ${ }^{14}$. Así podríamos eximirnos completamente de contestar preguntas sobre identidad y diferencia de objetos entre mundos posibles sin renunciar a que existan condiciones de verdad para los enunciados modales de re.

En todo caso, siempre resultará teóricamente preferible hallar una solución que, al mismo tiempo que respete la intuición central de que los hechos modales ${ }^{15}$ son hechos sobre propiedades -formas en que podrían ser las cosas- respete también nuestras intuiciones sobre possibilia indiscernibles. Una alternativa teórica podría ser reformular el universal de pluriverso del siguiente modo:

$$
\begin{aligned}
& \lambda y \diamond \exists x_{1} \diamond \exists x_{2} \ldots \diamond \exists x_{\mathrm{n}}\left[\left(( ( x _ { 1 } < y ) \wedge U _ { 1 } x _ { 1 } \wedge U _ { 2 } x _ { 1 } \wedge \ldots \wedge U _ { \mathrm { n } } x _ { 1 } ) \wedge \left(\left(x_{2}<y\right) \wedge U_{2} x_{2}\right.\right.\right. \\
& \left.\wedge U_{3} x_{2} \wedge \ldots \wedge U_{\mathrm{n}+1} x_{2}\right) \wedge \ldots\left(\left(x_{\mathrm{n}}<y\right) \wedge U_{\mathrm{n}} x_{\mathrm{n}} \wedge U_{\mathrm{n}+1} x_{\mathrm{n}} \wedge \ldots \wedge U_{\mathrm{n}+\mathrm{m}} x_{\mathrm{n}}\right) \vee\left(\left(\left(x_{1}<\right.\right.\right. \\
& \left.y) \wedge U_{2} x_{1} \wedge U_{3} x_{1} \wedge \ldots \wedge U_{\mathrm{n}+1} x_{1}\right) \wedge\left(\left(x_{2}<y\right) \wedge U_{3} x_{2} \wedge U_{4} x_{2} \wedge \ldots \wedge U_{\mathrm{n}+2} x_{2}\right) \wedge \ldots \\
& \left.\left(\left(x_{\mathrm{n}}<y\right) \wedge U_{\mathrm{n}} x_{\mathrm{n}} \wedge U_{\mathrm{n}+1} x_{\mathrm{n}} \wedge \ldots \wedge U_{\mathrm{m}} x_{\mathrm{n}}\right)\right) \vee \ldots \vee\left(\left(\left(x_{1}<y\right) \wedge U_{\mathrm{m}} x_{1} \wedge U_{\mathrm{m}+1} x_{1} \wedge \ldots\right.\right. \\
& \left.\wedge U_{\mathrm{m}+\mathrm{s}} x_{1}\right) \wedge\left(\left(x_{2}<y\right) \wedge U_{\mathrm{m}+1} x_{2} \wedge U_{\mathrm{m}+2} x_{2} \wedge \ldots \wedge U_{\mathrm{m}+\mathrm{q}} x_{2}\right) \wedge \ldots\left(\left(x_{\mathrm{n}}<y\right) \wedge U_{\mathrm{q}} x_{\mathrm{n}} \wedge\right. \\
& \left.\left.\left.U_{\mathrm{q}+1} x_{\mathrm{n}} \wedge \ldots \wedge U_{\mathrm{q}+\mathrm{s}} x_{\mathrm{n}}\right)\right)\right]
\end{aligned}
$$

14 No es necesario sostener una metafísica posibilista para admitir contrapartidas como forma de resolver los problemas de identidad y diferencia de objetos en diferentes mundos posibles. Hay, de hecho, filósofos actualistas que utilizan contrapartidas (cf. Sider 2002, pp. 301-304.)

15 Un "hecho modal" debe tomarse como el hecho de que es posible que esté nublado maña$n a$ o el hecho de que es necesario que $2+2=4$ o el hecho de que Juan es posiblemente sabio o Juan es necesariamente humano. 
En esta formulación del universal de pluriverso no resultará que deban postularse entidades $x_{1}, x_{2}, \ldots, x_{\mathrm{n}}$ que caigan bajo las propiedades estructurales especificadas en cada una de las cláusulas disyuntivas. Al estar ejemplificado el universal (UP') solo resultará que:

$$
\begin{aligned}
& \diamond \exists x_{1} \diamond \exists x_{2} \ldots \diamond \exists x_{\mathrm{n}}\left[\left(( U _ { 1 } x _ { 1 } \wedge U _ { 2 } x _ { 1 } \wedge \ldots \wedge U _ { \mathrm { n } } x _ { 1 } ) \wedge ( U _ { 2 } x _ { 2 } \wedge U _ { 3 } x _ { 2 } \wedge \ldots \wedge U _ { \mathrm { n } + 1 } x _ { 2 } ) \wedge \ldots \left(U_{\mathrm{n}} x_{\mathrm{n}} \wedge\right.\right.\right. \\
& \left.\left.U_{\mathrm{n}+1} x_{\mathrm{n}} \wedge \ldots \wedge U_{\mathrm{n}+\mathrm{m}} x_{\mathrm{n}}\right)\right) \vee\left(\left(U_{2} x_{1} \wedge U_{3} x_{1} \wedge \ldots \wedge U_{\mathrm{n}+1} x_{1}\right) \wedge\left(U_{3} x_{2} \wedge U_{4} x_{2} \wedge \ldots \wedge U_{\mathrm{n}+2} x_{2}\right) \wedge \ldots\right. \\
& \left(U_{\mathrm{n}} x_{\mathrm{n}} \wedge U_{\mathrm{n}+1} x_{\mathrm{n}} \wedge \ldots \wedge U_{\mathrm{m}} x_{\mathrm{n}}\right) \vee \ldots \vee\left(( U _ { \mathrm { m } } x _ { 1 } \wedge U _ { \mathrm { m } + 1 } x _ { 1 } \wedge \ldots \wedge U _ { \mathrm { m } + \mathrm { s } } x _ { 1 } ) \wedge \left(U_{\mathrm{m}+1} x_{2} \wedge U_{\mathrm{m}+2} x_{2}\right.\right. \\
& \left.\left.\wedge \ldots \wedge U_{\mathrm{m}+\mathrm{q}} x_{2}\right) \wedge \ldots\left(U_{\mathrm{q}} x_{\mathrm{n}} \wedge U_{\mathrm{q}+1} x_{\mathrm{n}} \wedge \ldots \wedge U_{\mathrm{q}+\mathrm{s}} x_{\mathrm{n}}\right)\right]
\end{aligned}
$$

Esto es, es posible que existan entidades $x_{1}, x_{2}, \ldots, x_{\mathrm{n}}$ que caerían, si existiesen, bajo las diferentes cláusulas disyuntivas. Esto es, intuitivamente, lo que el defensor de la concepción modal basada en universal quiere sostener. La dificultad que parece traer consigo esta estrategia es que deben utilizarse operadores modales de posibilidad antecediendo a los cuantificadores. Se supone que la motivación teórica fundamental de una metafísica modal actualista es explicar en qué consisten los hechos modales. Si esa explicación ha de utilizar, a su vez, nociones modales, entonces no parece una buena explicación. Se pretende explicar, por ejemplo, cómo es que " $p$ es posible" es verdadero si y solo si, $p$ es verdadera en algún mundo posible y, luego, que un "mundo posible" es nada más que un universal especialmente complejo. Si esta dilucidación de, por ejemplo, la posibilidad, debe en último término volver a hacer aparecer la misma noción de posibilidad, no parece claro qué dilucidación se ha efectuado (si se ha efectuado alguna).

Un recurso muy utilizado por diversos filósofos para enfrentar esta clase de dificultades ha sido negar que se pretenda hacer un análisis reductivo de la modalidad. Las nociones modales, tal como muchas otras, están sistemáticamente conectadas con otras, pero sin que puedan ser "eliminadas" mediante nociones más básicas que no sean ellas mismas de carácter moda ${ }^{16}$. Una teoría ontológica de la modalidad basada en universales habrá de explorar las conexiones sistemáticas que existen entre, por ejemplo, la modalidad y los universales, pero no debe pretender eliminar la modalidad por universales. El problema que ha surgido aquí es una muestra más de este tipo de conexiones, por lo que no debe sorprender que, finalmente, el espacio modal-tal como ordinariamente lo concebimos-solo pueda dilucidarse mediante el recurso ostensible de operadores modales. Esta es una cuestión delicada que no puede ser adjudicada aquí de manera sumaria. Probablemente solo podamos ponderar apropiadamente los costos y beneficios de una estrategia semejante cuando se alcance más claridad sobre cuáles son también los costos y beneficios de estrategias alternativas, tal como simplemente renunciar del todo a las identidades y diferencias de objetos entre diferentes mundos posibles. Esto deberá aguardar para una investigación ulterior.

16 Cf. por ejemplo, Plantinga (1974, pp. 44-45); Sider (2002, pp. 309-310). Una explicación general de cómo se hace uso en todas las formas de actualismo de nociones modales no analizadas en Divers (2002, pp. 181-195). 


\section{Conclusiones}

Se ha presentado en este trabajo la idea de un pluriverso como un refinamiento de la tesis de que los hechos modales tienen que ver con una pluralidad de mundos posibles. Primero se ha explicado la motivación y la configuración de una "oración de pluriverso" para el caso de la teoría modal lingüística, y luego se ha explicado cómo es que esta idea general puede ser trasladada para el caso de una concepción modal basada en universales, en la que los mundos posibles deberían ser entendidos como universales estructurales máximos.

El universal de pluriverso, tal como se ha explicado arriba, parece poder representar cómo es que pueden darse dos o más objetos posibles no actuales indiscernibles por lo que respecta a qué propiedades poseen y cómo es que luego exactamente uno de tales objetos podría existir sin el segundo. En el universal de pluriverso las formas en que podría ser el mundo quedan representadas como cláusulas disyuntivas. El universal de pluriverso, entonces, es una gran propiedad que determina el espacio modal completo y que, en algún sentido, podría ser denominada la "esencia del mundo". Se explica aquí, luego, cómo es que los análisis clásicos de las nociones modales pueden ser preservados como indicaciones de lo que acaecería si cláusulas disyuntivas de (UP) fuesen ejemplificadas.

La dificultad fundamental de (UP), sin embargo, dejando a un lado las dudas generales que pudiesen existir sobre universales trascendentes, estructurales, disyuntivos, etc., es que pareciera exigir que estuviese dado ya de entrada un dominio de entidades actuales y meramente posibles sobre la que se debería estar cuantificando. Esto iría directamente en contra de las motivaciones iniciales para preferir una explicación de los hechos modales basada en universales. No es claro, sin embargo, cómo esta dificultad podría ser resuelta ${ }^{17}$.

17 Este trabajo ha sido redactado en ejecución del proyecto de investigación Fondecyt 1070339 (Conicyt, Chile), durante una estadía de investigación en la Facultad de Filosofía de la Universidad de Oxford (Reino Unido). Agradezco las facilidades entregadas por las autoridades de la Facultad para mi trabajo. Una versión preliminar de este escrito fue presentada en el seminario del GAF, Facultad de Filosofía, Universidad de Buenos Aires (Argentina) y en el II Coloquio Internacional de la Sociedad Chilena de Filosofía Analítica (Santiago de Chile, 8 al 10 de octubre de 2008) Agradezco los comentarios de los asistentes en ambas ocasiones y también las útiles sugerencias y correcciones de un evaluador anónimo de esta revista. 
Referencias bibliográficas

Adams, Robert M. (1979), "Primitive Thisness and Primitive Identity". The Journal of Philosophy. 76: 5-26.

Alvarado, José Tomás (2007), "Esencias individuales e identidad primitiva". Analytica 11: $155-195$.

Armstrong, David M. (1978a), Universals and Scientific Realism, Volume I, Nominalism and Realism. Cambridge: Cambridge University Press.

(1978b), Universals and Scientific Realism. Volume II. A Theory of Universals. Cambridge: Cambridge University Press.

(1986), "In Defence of Structural Universals". Australasian Journal of Philosophy. 64: 85-88.

(1989), Universals. An Opinionated Introduction. Boulder: Westview. Press. (1997), A World of States of Affairs. Cambridge: Cambridge University

Bennett, Jonathan (2003), A Philosophical Guide to Conditionals. Oxford: Clarendon Press.

Bigelow, John (1986), "Towards Structural Universals". Australasian Journal of Philosophy. 64: 94-96.

Bigelow, John y Robert Pargetter (1990), Science and Necessity. Cambridge: Cambridge University Press.

Black, Max (1999), "The Identity of Indiscernibles", en J. Kim y E. Sosa, eds., Metaphysics. An Anthology. Oxford: Blackwell, pp. 66-71.

Divers, John (2002), Possible Worlds. London: Routledge.

Forrest, Peter (1986a), "Ways Worlds Could Be". Australasian Journal of Philosophy. 64: $15-24$.

(1986b), "Neither Magic Nor Mereology: A Reply to Lewis". Australasian Journal of Philosophy. 64: 89-91.

Lewis, David (1973), Counterfactuals. Oxford: Blackwell.

(1983), "Counterpart Theory and Quantified Modal Logic", en Philosophical Papers. Volume I. Oxford: Oxford University Press, pp. 26-46.

(1986a), On the Plurality of Worlds. Oxford: Blackwell.

(1986b), "Against Structural Universals". Australasian Journal of Philosophy. 64: 25-46.

(1986c), "Comment on Armstrong and Forrest". Australasian Journal of Philosophy. 64: 92-93.

Melia, Joseph (2003), Modality. Chesham: Acumen.

Plantinga, Alvin (1974), The Nature of Necessity. Oxford: Clarendon Press.

Sider, Theodor (2002), "The Ersatz Pluriverse”. The Journal of Philosophy. 99: 279315. 
Stalnaker, Robert C. (2003), "Possible Worlds", en Ways a World Might Be, Oxford: Clarendon Press, pp. 25-39. Originalmente publicado en Noûs 10 (1976).

Williamson, Timothy (2007), The Philosophy of Philosophy. Oxford: Blackwell. 